\title{
Watching Sex on Television Predicts Adolescent Initiation of Sexual Behavior
}

\author{
Rebecca L. Collins, PhD*; Marc N. Elliott, PhD*; Sandra H. Berry, MA*; David E. Kanouse, PhD*; \\ Dale Kunkel, PhD¥; Sarah B. Hunter, PhD*; and Angela Miu, MS*
}

\begin{abstract}
Background. Early sexual initiation is an important social and health issue. A recent survey suggested that most sexually experienced teens wish they had waited longer to have intercourse; other data indicate that unplanned pregnancies and sexually transmitted diseases are more common among those who begin sexual activity earlier. The American Academy of Pediatrics has suggested that portrayals of sex on entertainment television (TV) may contribute to precocious adolescent sex. Approximately two-thirds of TV programs contain sexual content. However, empirical data examining the relationships between exposure to sex on TV and adolescent sexual behaviors are rare and inadequate for addressing the issue of causal effects.

Design and Participants. We conducted a national longitudinal survey of 1792 adolescents, 12 to 17 years of age. In baseline and 1-year follow-up interviews, participants reported their TV viewing habits and sexual experience and responded to measures of more than a dozen factors known to be associated with adolescent sexual initiation. TV viewing data were combined with the results of a scientific analysis of TV sexual content to derive measures of exposure to sexual content, depictions of sexual risks or safety, and depictions of sexual behavior (versus talk about sex but no behavior).

Outcome Measures. Initiation of intercourse and advancement in noncoital sexual activity level, during a 1-year period.
\end{abstract}

Results. Multivariate regression analysis indicated that adolescents who viewed more sexual content at baseline were more likely to initiate intercourse and progress to more advanced noncoital sexual activities during the subsequent year, controlling for respondent characteristics that might otherwise explain these relationships. The size of the adjusted intercourse effect was such that youths in the 90th percentile of TV sex viewing had a predicted probability of intercourse initiation that was approximately double that of youths in the 10th percentile, for all ages studied. Exposure to TV that included only talk about sex was associated with the same risks as exposure to TV that depicted sexual behavior. African American youths who watched more depictions of sexual risks or safety were less likely to initiate intercourse in the subsequent year.

Conclusions. Watching sex on TV predicts and may hasten adolescent sexual initiation. Reducing the amount of sexual content in entertainment programming, reduc-

From *RAND, Santa Monica, California; and $\ddagger$ University of California, Santa Barbara, California.

Accepted for publication Apr 1, 2004.

DOI: $10.1542 /$ peds.2003-1065-L

Reprint requests to (R.L.C.) RAND, 1700 Main St, Santa Monica, CA 90407. E-mail: collins@rand.org

PEDIATRICS (ISSN 0031 4005). Copyright (C) 2004 by the American Academy of Pediatrics. ing adolescent exposure to this content, or increasing references to and depictions of possible negative consequences of sexual activity could appreciably delay the initiation of coital and noncoital activities. Alternatively, parents may be able to reduce the effects of sexual content by watching TV with their teenaged children and discussing their own beliefs about sex and the behaviors portrayed. Pediatricians should encourage these family discussions. Pediatrics 2004;114:e280-e289. URL: http: //www.pediatrics.org/cgi/content/full/114/3/e280; condoms, media, sex, television.

$\overline{\text { ABBREVIATIONS. STD, sexually transmitted disease; TV, tele- }}$ vision.

$\Lambda$ key period of sexual exploration and development occurs during adolescence. During this time, individuals begin to consider which sexual behaviors are enjoyable, moral, and appropriate for their age group. ${ }^{1}$ Many teens become sexually active during this period; currently, $46 \%$ of high school students in the United States have had sexual intercourse. ${ }^{2}$ Although intercourse among youths is common, most sexually active teens wish they had waited longer to have sex, ${ }^{3}$ which suggests that sex is occurring before youths are prepared for its consequences. Additional evidence of this is provided by public health data. Each year, 1 case of a sexually transmitted disease (STD) is diagnosed for every 4 sexually active teens in the United States, ${ }^{4}$ and the US rate of teen pregnancy is among the highest of all industrialized countries. ${ }^{5}$ Unplanned pregnancies and STDs are more common among those who begin sexual activity earlier. ${ }^{6}$

Therefore, early sexual initiation is an important health issue. This raises the question of why individuals become sexually involved at younger ages. What factors hasten sexual initiation, and what factors delay its onset? There are many well-documented predictors of age of intercourse initiation, both social and physical. However, 1 factor commonly cited by parents and policy makers as promoting sex among teens has received little systematic scientific investigation, namely, television (TV). There is good scientific reason to think that TV may be a key contributor to early sexual activity. Sexual behavior is strongly influenced by culture, 7,8 and TV is an integral part of US teen culture. The average youth watches $\sim 3$ hours of TV daily. ${ }^{9}$ There, sexual messages are commonplace, according to a scientific content analysis of a representative sample of pro- 
gramming from the 2001-2002 TV season. Sexual content appears in $64 \%$ of all TV programs; those programs with sexual content average 4.4 scenes with sexually related material per hour. Talk about sex is found more frequently (61\% of all programs) than overt portrayals of any sexual behavior $(32 \%$ of programs). Approximately 1 of every 7 programs $(14 \%)$ includes a portrayal of sexual intercourse, depicted or strongly implied. ${ }^{10}$

This high-dose exposure to portrayals of sex may affect adolescents' developing beliefs about cultural norms. TV may create the illusion that sex is more central to daily life than it truly is and may promote sexual initiation as a result, a process known as media cultivation. ${ }^{11}$ Exposure to the social models provided by TV also may alter beliefs about the likely outcome of engaging in sexual activity. Social learning theory predicts that teens who see characters having casual sex without experiencing negative consequences will be more likely to adopt the behaviors portrayed. ${ }^{12}$ Although televised sexual portrayals can theoretically inhibit sexual activity when they include depictions of sexual risks (such as the possibility of contracting an STD or becoming pregnant), abstinence, or the need for sexual safety, this type of depiction occurs in only $15 \%$ of shows with sexual content. In other words, only $\sim 1$ of every 7 TV shows that include sexual content includes any safe sex messages, and nearly two-thirds of these instances $(63 \%)$ are minor or inconsequential in their degree of emphasis within the scene. ${ }^{10}$ As a result, sexual content on TV is far more likely to promote sexual activity among US adolescents than it is to discourage it. TV has already been shown to influence violent and aggressive behavior among youths ${ }^{13}$ and, although extension of the principles involved to the realm of sexual behavior is not a foregone conclusion, the hypothesis that TV promotes early sexual initiation logically follows from previous media effects theory and several existing studies.

Previous work demonstrated links between viewing of sexual content on TV and attitudes toward sex, ${ }^{14}$ endorsement of gender stereotypes likely to promote sexual initiation, ${ }^{15}$ and dissatisfaction with virginity, ${ }^{16}$ as well as a wide range of perceptions regarding normative sexual behavior. ${ }^{17}$ In addition to these studies, 2 groundbreaking articles published in the early 1990s examined the question of whether exposure to sex on TV influences adolescent sexual behavior. The studies found positive associations between any lifetime intercourse and TV viewing among adolescents, but methodologic limitations rendered the results inconclusive. ${ }^{18,19}$ Higher levels of exposure to sex on TV might have led to sexual initiation in that research, but a plausible alternative interpretation was that, because sexual content more closely reflects their identities and interests, sexually active adolescents choose to watch more of it than their inactive peers. This possibility could not be excluded, because the relative timing of these events was unknown to the researchers. These 2 studies were also limited in their ability to attribute the sexual behavior of youths to differences in TV exposure to sex, rather than to other closely related fac- tors. For example, youths who receive little supervision may be free to watch more TV and to choose programs with sexual content and may also have more opportunities to engage in sexual activity. Finally, previous work was forced to rely on imprecise measures of content, making it difficult to be certain that exposure to sexual content per se was the source of the associations observed.

Here we describe the results of a study designed to test the effects of TV sexual content on adolescent sexual initiation with more stringent methods. We examined the effects of exposure to TV sexual content overall, exposure to TV depictions of sexual risks or sexual safety, and exposure to TV portrayals of sexual behavior versus talk about sex. Although TV producers and the general public have expressed concern regarding both sexual talk and sexual behavior, ${ }^{20}$ portrayals of behavior have typically been the focus of such attention. Given the potential applications of our research, we considered it important to determine whether this emphasis is well placed. Social learning theory predicts that observation of either sexual talk or sexual behavior will influence teens to have sex, as long as the portrayed consequences are not negative, but the theory does not address whether the magnitudes of these effects will differ. ${ }^{12}$ Finally, we examined the effects of hours spent viewing TV, independent of content. Some theory and research argue that any time spent watching TV affects sexual behavior, ${ }^{11,19}$ whereas other research suggests that only programs with known sexual content have an influence. ${ }^{12,18}$ The issue warrants examination, because the implications of the 2 results, with respect to theory, programming, policy, and parenting, are quite different.

We collected longitudinal data, conducting 2 surveys 1 year apart. TV viewing was assessed at baseline and was used to predict initiation of sexual activities in the following year among 12- to 17-yearold subjects, taking into account sexual experience at baseline. This design allowed for the possibility that sexual experience influences teens' selection of sexual TV content and accounted for the possibility in testing for the effects of TV on sexual behavior. The design also took into account variables that might contribute to both sexual behavior and exposure to sexual content on TV, creating a spurious relationship between the 2. Statistically controlling for a large set of differences between youths with high and low exposure to sex on TV in this study enabled us to attribute more confidently any differences in their sexual behavior to their TV viewing habits. A final methodologic strength was our use of detailed reliable measures of various forms of sexual content. With the increasing diversity of TV programming, this has become essential for assessment of contentspecific effects of viewing. These precise measures also enabled more sensitive tests of TV effects, compared with those possible in previous studies.

We hypothesized that adolescents exposed to greater amounts of sexual content on TV would initiate intercourse sooner and would progress more quickly to higher levels of noncoital activity. However, we expected that exposure to portrayals of 
sexual safety or the risks that accompany sexual activity would be associated with a delay in sexual advancement. Tests regarding the effects of exposure to sexual behavior relative to talk about sex were exploratory, as were tests to determine whether hours of TV viewing were related to sexual behavior changes. Therefore, we made no predictions regarding those 2 variables.

\section{METHODS}

\section{Procedure}

We conducted a national telephone survey in spring 2001 and reinterviewed the same group 1 year later, in spring 2002. The survey measured TV viewing habits, sexual knowledge, attitudes, and behavior, and a large set of demographic and psychosocial variables shown to predict sexual behavior or TV viewing habits in previous research.

A list of households with a high estimated probability $(37.5 \%)$ of containing a 12- to 17-year-old adolescent was purchased from Survey Sampling Inc. The list was developed with information from residential telephone listings, supplemented with data from the Department of Motor Vehicles (driver's license and car registration data), magazine subscriptions, product warranty registrations, and school enrollment records. To be eligible for the list, the household address had to be available from 1 of these sources. In an effort to obtain representative numbers of minority participants, who tend to be underrepresented on such lists, households from census tracts with $>30 \%$ minority residents (African American, Asian American, or Hispanic) were oversampled by a factor of 3.25 .

Enrolling adolescents in a telephone survey that assesses sexual behavior requires care and sensitivity. All households were sent a letter describing the study before telephone contact, so that they could carefully consider their participation. At the time of the baseline telephone interview, we briefly surveyed the parents, to determine the household composition and to measure the parents' sexual attitudes. An adolescent participant was then randomly selected from among all household members in the age range of 12 to 17 years. Parental consent for the adolescent's participation and then the adolescent's assent were obtained before the interview. Although we suggested that adolescents speak to us in a room where they could respond to the interview privately, we enhanced confidentiality and promoted honest responding by asking questions in such a way that anyone listening to the adolescent's side of the conversation would be unable to link his or her responses (eg, "yes" or "most of the time") to item content.

\section{Sample}

We obtained an overall completion rate at baseline of $54 \%$ (n[r] $=2003$ ). The refusal rate was $36 \%$ among households determined to be eligible, consistent with the results of telephone surveys on less-sensitive topics. At the follow-up interviews, we retained $88 \%$ of the baseline sample, resulting in a final sample of 1762 adolescents, 12 to 17 years of age, who participated in both interviews. This longitudinal sample was $48 \%$ female, $77 \%$ white, $13 \%$ African American, $7 \%$ Hispanic, and $4 \%$ Asian or other race. At least 1 parent had a college degree for $33 \%$ of the sample; $64 \%$ had a parent who had been educated otherwise beyond high school. Seventeen percent had ever had intercourse at baseline and 29\% at the follow-up assessments. Among adolescents 15 to 17 years of age (for whom comparable data are available), the percentages who reported ever having had intercourse in the follow-up interviews ( $40 \%$ of boys and $32 \%$ of girls) were similar to those found in other national surveys with household sampling frames, such as the National Survey of Adolescent Males and the National Survey of Family Growth. ${ }^{21}$

\section{Measures}

\section{Exposure to Sexual Content on TV}

Three measures reflected the content of TV viewed at baseline, ie, exposure to sexual content, exposure to portrayals of sexual risks or the need for safety, and relative exposure to sexual behavior versus talk about sex. These measures were based on a set of 23 programs. To generate the program list, we used Nielsen ratings of the regularly scheduled prime time programs most watched by 4 groups (male and female 12-14-year-olds and 15-17year-olds), in the period from October 2, 2000, to February 18, 2001 (the fall season up until our survey field period began). Eligible programs appeared on ABC, NBC, CBS, Fox, WB, UPN, or PAX. After eliminating movies, sports, game shows, and specials (1time airings), we included 15 of the 20 programs on the top 10 list for 1 of the 4 groups. Five additional broadcast programs and 3 cable programs that were known (on the basis of prior content analyses) or expected (on the basis of reviews in the popular press) to contain high levels of sexual content were also included in the list. The final list included programs appearing on broadcast networks and basic and premium cable channels and encompassed animated and live action shows, reality shows, sitcoms, and dramas. As part of the baseline survey, teens indicated the frequency with which they watched these 23 programs during the previous TV season ("since school started last fall") on a 4-point scale, ranging from "never" to "every time it's on." We derived the exposure measures by multiplying the self-reported viewing frequency for each program by 1 of 3 indicators of the average content in an episode of that program and summing across programs.

Methods developed by Kunkel et al, ${ }^{10}$ as part of a much larger study of TV sexual content, were used to determine the sexual content in a sample of episodes for the 23 programs. A minimum of 3 episodes and a maximum of 14 were taped and coded for each program. We used tapes from the random TV sample for the larger study and thus had variable numbers of episodes available for each program. In cases in which there were $<3$ episodes in the library for the larger study, we taped additional episodes. Technical difficulties (discovered after the TV season) resulted in the use of only 2 episodes for 2 programs studied. Coders unitized the episodes into distinct scenes, indicating the presence of any of the following: 1) sexual behavior: physical flirting, passionate kissing, intimate touch, intercourse implied, or intercourse depicted; 2) sexual talk: talk about own/others' plans or desires, talk about sex that has occurred, talk toward sex, expert advice, or other; or 3) talk or behavior depicting risks or the need for safety in regard to sexual activity: abstinence, waiting to have sex, portrayals mentioning or showing condoms or birth control, and portrayals related to acquired immunodeficiency syndrome, STDs, pregnancy, or abortion. These 3 categories of content were not exclusive of one another, and a given scene could contain all or none of these broad categories. Raters also coded the degree of focus (major or minor) on sexual behavior, talk, or risks in each scene. Highly trained and experienced raters from the larger study by Kunkel et $\mathrm{al}^{10}$ coded our data. Inter-rater reliabilities for the larger study ranged from 89 to $100 \%$ for the sexual content variables used in the present study. ${ }^{10}$

For each TV series studied, the amount of sexual content was calculated as the average number of scenes per episode containing a major focus on sexual behavior plus the average number of scenes containing a major focus on talk about sex. Initial analyses indicated that the effects of exposure to sexual talk and sexual behavior in the same scene were additive, rather than redundant. Similar analyses indicated that no predictive information was gained by weighting shows according to length (30 minutes vs 1 hour). We also found that including measures of exposure to scenes with a minor focus on sexual behavior or sexual talk in our equations did not add to prediction. The proportion of sexual content that included sexual behavior was measured by dividing the average number of scenes that contained a major focus on sexual behavior by the average number of scenes with any sexual content for each episode. Risk and safety content was calculated as the average number of scenes per episode containing any such portrayal, whether the focus was major or minor. Because sexual risks and the need for safety were rarely portrayed, using only scenes with a major focus would have caused the risk measure to focus unduly on only a few programs and episodes.

With weighting according to viewing frequency and summing across programs, the 3 measures exhibited generally good distributional properties. All were standardized to a mean of 0 and SD of 1 . Scree plots indicated that exposure to sexual content and exposure to risk and safety content were somewhat positively skewed (ranges: -1.43 to 4.49 and -0.98 to 4.97 , respectively), whereas the sexual behavior-to-total sex proportion measure was more normally distributed (range: -2.82 to 1.68 ). 


\section{Average Hours of TV Viewing}

We measured time spent watching TV with a set of 5 items assessing hours of viewing on various days of the week and at different times of day. Responses were averaged to create a continuous indicator of average viewing time $(\alpha=.70)$.

\section{Sexual Behavior}

Questions assessed behavior with someone of the opposite sex. Intercourse experience at both the baseline and follow-up assessments was measured with the item "Have you ever had sex with a boy/girl? By sex we mean when a boy puts his penis in a girl's vagina" (yes/no). Twelve adolescents who reported intercourse experience at the baseline assessment but not at the follow-up assessment were classified as having had intercourse at both time points. We measured lifetime levels of noncoital experience with a scale developed for this study, based on a measure used by Miller et al. ${ }^{22}$ Adolescents indicated whether they had ever 1) kissed,*2) "made-out" (kissed for a long time), 3) touched a breast/had their breast touched, ${ }^{*} 4$ ) touched genitals/had their genitals touched, or 5 ) given or received oral sex. Items with an asterisk were asked of all youths, and the others were asked only if the response to the item listed immediately before it was yes. Participants received scores of 1 to 5 , reflecting the highest level of behavior experienced; adolescents who reported none of the noncoital behaviors were included in the lowest category, along with those who had only kissed.

\section{Covariates}

Covariates were measured as part of the baseline interview. Gender and race/ethnicity were self-reported. Respondent age was measured continuously, in years, and was calculated from the date of birth and the baseline interview date.

We included several indicators of social environment known to predict initiation of coitus. A single item assessed whether the respondent's friends were primarily older, younger, or about the respondent's age and was dichotomized to indicate older versus all other responses. Teens who reported living with both of their parents were classified as such (versus all others). Parent education was measured as schooling completed by the more highly educated parent, using a 6-point scale ( $1=$ less than high school; $6=$ completed graduate or professional degree). Parental monitoring was assessed with a 5-item measure (items rated from $1=$ strongly agree to $5=$ strongly disagree) developed to predict adolescent risk behavior $(\alpha=.68) .{ }^{23}$ Teens also indicated their mothers' work status outside the home $(1=$ none; 2 = part-time; $3=$ full-time). An additional measure assessed parental prescriptive norms, by asking the perceived parental response if the respondent had sex in the following year. The response options ranged from 1 ("disapprove a lot") to 5 ("approve a lot"). Because responses were bimodal, we recoded the item to dichotomously reflect parent disapproval (responses of 1 or 2 ) versus approval or neutrality (responses of 3, 4, or 5).

Lower educational expectations have been shown to predict early childbearing ${ }^{24}$ and may affect adolescent sexual risk-taking more broadly. We recorded 2 measures related to this. Respondents self-reported their grades in school $(1=$ mostly As; $2=$ mostly Bs; $3=$ mostly Cs; $4=$ mostly Ds; $5=$ mostly Fs). Adolescents' educational aspirations were assessed with an item that asked, "What is the highest level of school you plan to finish?" $(1=$ less than college; 2 = college; 3 = graduate or professional school).

We also assessed a number of other personal characteristics that are known correlates of adolescent sexual behavior. Mental health $(\alpha=.68)$ was assessed with the Mental Health Index 5, a wellvalidated, 5 -item scale assessing affective states during the previous 4 weeks. ${ }^{25}$ Self-esteem was measured with 3 items from the Rosenberg Self-Esteem Inventory $(\alpha=.72) .{ }^{26}$ For measurement of religiosity, respondents indicated their agreement that "religion is very important in my life," on a 4-point scale. Deviant behavior was assessed with a 6-item measure drawn from prior studies of adolescent risk behavior $(\alpha=.62) .{ }^{27}$ Respondents indicated how many times in the past 12 months they had been sent out of class, broken into a house, school, or place of business, skipped school, cheated on a test, damaged something on purpose, or stolen something ( $1=$ not at all; $4=\geq 10$ times). Sensation-seeking was measured with 3 items from the Zuckerman ${ }^{28}$ scale that had high factor loadings in a previous study and contained content appropriate to adolescents $(\alpha=.57)$.

\section{Analyses}

\section{Preliminary Analyses}

One hundred seventy-five respondents declined to answer some or all of the sexual behavior questions and were excluded from analyses. A multivariate logistic regression analysis compared these individuals with the sample who responded to the sex questions. Predictors tested were age, gender, race, TV viewing, TV viewing involvement, exposure to sexual TV, religiosity, and believing that most kids have had sex by the time they reach the respondent's age. The results indicated that youths who skipped the sex questions were heavier and more-involved TV viewers and were more often African American (than white) $(P<.05)$. They watched marginally less sex on TV $(P=.10)$. To control for sexual behavior at baseline, the analysis sample for predicting intercourse was restricted to baseline virgins with complete data on sexual behavior $(n=1292)$. Tests predicting noncoital behavior used all subjects with data on sexual behavior $(n=1581)$ but controlled for the level of noncoital behavior at baseline. We used logistic regression analyses to predict intercourse and ordinal logistic regression analyses to predict levels of noncoital behavior in all tests.

Our preliminary analyses tested simple associations between the baseline TV viewing variables and sexual behavior. Because youths who see more sex on TV are also youths who watch more TV overall and who therefore see more sexual risk and safety content, the TV variables are best understood in the context of one another. We thus examined all $4 \mathrm{TV}$ variables simultaneously in these tests. We also tested whether other respondent characteristics might explain any relationship between viewing sexual content and behavior, by examining bivariate associations between these characteristics and sex-heavy TV viewing at the baseline evaluation, intercourse initiation by the follow-up interview, and advancement in the level of noncoital behavior by the follow-up interview.

Our key analyses were a pair of multivariate regression equations including all TV viewing variables and all covariates as predictors of changes in sexual behavior from the baseline assessment to the follow-up assessment. A final pair of equations incorporated interaction terms, to test for differences in the associations between TV exposure and sexual behavior change as a function of age ( $<15$ years vs $\geq 15$ years of age), gender (male versus female), and race/ethnicity. Initial analyses showed no differences in TV effects for Hispanics versus non-Hispanic whites/others. Therefore, we compared African Americans with all other races in these models.

\section{Sampling and Nonresponse Weights}

Without weights, sample demographic characteristics were generally representative of those of adolescents in the United States, but the group included somewhat fewer Hispanics and highly educated parents than expected. A multivariate logistic regression analysis predicting attrition on the basis of 32 baseline characteristics was nonsignificant, indicating no bias in our sample attributable to this factor. A similar analysis predicting nonresponse at baseline from information provided by the supplier of our sample and a brief nonresponse interview with parents identified 3 predictors of failure to enroll in the study. There was a trend toward higher response rates in tracts with higher proportions of African Americans and among households in which an adolescent 12 to 14 years of age was present but not randomly selected. We also obtained lower response rates when a boy $>14$ years of age was randomly selected for sampling. We created nonresponse weights inversely proportional to the probability of enrollment, as indicated by this equation. After application of these weights, there was still a slight sample bias, relative to US population statistics. Therefore, we also created poststratification weights, to bring our data more closely in line with US figures. ${ }^{29}$ Table 1 shows that the combined weights corrected the racial bias and adjusted other key sample characteristics. Therefore, all subsequent analyses used these weights.

\section{Imputation}

A small number of respondents had missing data for $\geq 1$ predictor variables. Although the percentage missing for any given 
TABLE 1. Effect of Weights on Baseline Sample Characteristics $(n=2003)$

\begin{tabular}{lcc}
\hline \multicolumn{1}{c}{ Characteristic } & \multicolumn{2}{c}{ Proportion, \% } \\
\cline { 2 - 3 } & Unweighted & Weighted \\
\hline Non-Hispanic white & 75 & 66 \\
Non-Hispanic black & 13 & 14 \\
Hispanic & 8 & 13 \\
Other race & 4 & 6 \\
Parent education less than & 2 & 9 \\
$\quad$ high school & 20 & 25 \\
Grades mostly C or lower* & 89 & 88 \\
Parents disapprove of sex & & 5 \\
Ever had sex* & 4 & 32 \\
$\quad$ 12-14 y of age & 27 & \\
15-17 y of age &
\end{tabular}

Poststratification and nonresponse weights were both used.

* Not used in the construction of poststratification or nonresponse weights but provided as illustrative of weighting effects.

variable was $<3 \%$, listwise deletion of cases would have resulted in significant sample loss in our main multivariate analyses. To avoid any bias this might introduce in our results, we imputed missing data for these predictors. ${ }^{30}$ Viewership responses for 2 programs had a total of 9 missing values. These were imputed with random draws from the empirical distribution. In addition, the first 263 teens interviewed were not asked about viewership of 4 programs, as a result of a programming error. The resulting missing values were imputed via random draws from the predicted probabilities of an ordinal logistic regression model, with age, gender, interaction between age and gender, average TV viewing time, and viewing frequency for the shows with no missing values serving as independent variables. A similar process, using all nonmissing covariates in the multivariate model as independent variables, was used to impute missing values for covariates.

\section{RESULTS}

Before controlling for other variables, a diet of TV high in sexual content at baseline was strongly related to initiation of intercourse and advancement of noncoital activity levels in the following year (Table 2). TV exposure to relatively more depictions of sexual behavior than talk were unrelated to either behavior, and although higher levels of overall viewing were negatively correlated with sexual advancement, these relationships did not reach statistical significance. However, TV exposure to the risks of sex was related to less progression in noncoital behavior.

Simple associations between other respondent characteristics and sexual activity during the following year are also displayed in Table 2 . As can be seen, many of these factors were strongly related to sexual behavior. The factors that were positively associated with initiation of intercourse among virgins were older age, having older friends, getting low grades, engaging in deviant behavior, and sensation-seeking. Those associated with a lower probability of intercourse initiation were parental monitoring, parent education, living with both parents, having parents

TABLE 2. Bivariate Associations Between Baseline Predictors, Exposure to Sex on TV at Baseline, and Sexual Behavior Change by Wave 2

\begin{tabular}{|c|c|c|c|}
\hline \multirow[t]{2}{*}{ Baseline Predictor } & \multicolumn{3}{|c|}{ Regression Coefficients } \\
\hline & $\begin{array}{l}\text { Baseline Exposure } \\
\text { to Sexual Content } \\
\quad(n=1762)^{*}\end{array}$ & $\begin{array}{l}\text { Intercourse } \\
\text { Initiation } \\
(n=1292)\end{array}$ & $\begin{array}{l}\text { Advancing Level } \\
\text { of Noncoital Sex } \\
\quad(n=1581) \dagger\end{array}$ \\
\hline \multicolumn{4}{|l|}{ TV exposuref } \\
\hline Sexual content & & $.47 \S$ & $.37 \S$ \\
\hline Sexual risk or need for safety & & -.19 & $-.21 \|$ \\
\hline Sexual behavior versus talk & & -.02 & -.01 \\
\hline Average hours TV viewing $\ddagger$ & & -.07 & -.12 \\
\hline \multicolumn{4}{|l|}{ Covariates } \\
\hline Age, yII & $.04 \|$ & $.39 \S$ & $.20 \S$ \\
\hline Female genderII & .04 & -.27 & -.22 \\
\hline Hispanic $\llbracket \#$ & -.14 & -.10 & -.23 \\
\hline African American II\# & $.25 \|$ & .19 & -.06 \\
\hline Has mostly older friends II & $-.18 \|$ & $.74 \S$ & .15 \\
\hline Lives with both parents II & -.06 & $-.54 \|$ & -.01 \\
\hline High parent education & -.05 & $-.33 \S$ & -.11 \\
\hline Parental monitoring & $-.14 \S$ & $-.57 \S$ & $-.33 \S$ \\
\hline Time mother works outside home & $.07 \|$ & .17 & .07 \\
\hline Parent disapproval of sexII & .00 & $-1.03 \S$ & -.03 \\
\hline Low school grades & $.11 \S$ & $.48 \S$ & $.24 \S$ \\
\hline High educational aspirations & $.06 \|$ & -.12 & -.08 \\
\hline Religious & $-.09 \S$ & $-.34 \S$ & $-.20 \S$ \\
\hline Good mental health & $-.08 \S$ & $-.38 \S$ & $-.19 \S$ \\
\hline Self-esteem & .01 & -.03 & -.10 \\
\hline Sensation-seeking & $.16 \S$ & $.60 \S$ & $.21 \S$ \\
\hline Deviant behavior & $.20 \S$ & $.52 \S$ & $.31 \S$ \\
\hline
\end{tabular}

* Total viewing hours were controlled statistically; therefore, the variable reflects viewing a relatively high amount of sexual content relative to other viewing.

t Predicts level of noncoital behavior at wave 2, using baseline level as a covariate.

$\ddagger$ The 4 television variables were examined as a group; therefore, partial correlations are reported.

$\S P<.01$.

$\| P<.05$.

II Age and dichotomous variables were not standardized because their scales are interpretable in nonstandardized form.

\# The comparison group was non-Hispanic white and races other than Hispanic and African American. 
who would disapprove if the adolescent had sex, being religious, and having good mental health. Only 1 additional variable predicted changes in noncoital activity; exposure to risk or safety portrayals was related to a lower level of noncoital activity. Variables that were not predictive of either outcome were gender, race, self-esteem, educational aspirations, and mother's work outside the home.

As can be seen in Table 2, most of the variables predictive of later sex were also correlated at baseline with a sex-heavy TV diet, and most correlations were in the same direction as those observed for sexual activity. These variables could potentially account for the relationships between exposure to sexual content and sexual activity observed. To test for TV effects independent of such factors, we entered all of the bivariate predictors of intercourse initiation or noncoital stage into our models as covariates. We also included the gender and race variables in multivariate analyses, because they were central to planned tests for subgroup differences. The resulting models were excellent predictors of the outcome variables. A concordance or $c$ statistic, ${ }^{31}$ scaled so that $50 \%$ corresponds to chance and $100 \%$ corresponds to perfect prediction, indicated that respondents' initiation of intercourse was correctly predicted $79 \%$ of the time and their advances in noncoital behavior were correctly predicted $90 \%$ of the time. Results are presented in Table 3.

The significant coefficient for sexual content in these models indicates that, after more than a dozen

TABLE 3. Multivariate Regression Equations Predicting Sexual Behavior at Wave 2 From Baseline TV Viewing and Other Characteristics

\begin{tabular}{|c|c|c|}
\hline \multirow[t]{2}{*}{ Baseline Predictor } & \multicolumn{2}{|c|}{$\begin{array}{l}\text { Logistic Regression } \\
\text { Coefficients }\end{array}$} \\
\hline & $\begin{array}{l}\text { Intercourse } \\
(n=1292)\end{array}$ & $\begin{array}{l}\text { Noncoital Sex } \\
(n=1581)\end{array}$ \\
\hline \multicolumn{3}{|l|}{ TV exposure } \\
\hline Sexual content & $.32 *$ & $.31 \dagger$ \\
\hline Sexual risk or need for safety & -.18 & -.17 \\
\hline Sexual behavior versus talk & .11 & .04 \\
\hline Total hours TV viewing & .04 & -.10 \\
\hline \multicolumn{3}{|l|}{ Covariates } \\
\hline Age, ył & $.42+$ & $.22+$ \\
\hline Female gender $\ddagger$ & -.16 & -.15 \\
\hline Hispanicłミ & -.50 & -.37 \\
\hline African American $\ddagger \S$ & .40 & .02 \\
\hline Has mostly older friends $\ddagger$ & $.69^{*}$ & .15 \\
\hline Lives with both parents $\ddagger^{\top}$ & $-.58^{*}$ & -.01 \\
\hline High parent education & $-.31+$ & -.13 \\
\hline Parental monitoring & $-.25^{*}$ & $-.17^{*}$ \\
\hline Parent disapproval of sex $\ddagger$ & -.37 & .13 \\
\hline Low school grades & $.27^{*}$ & .15 \\
\hline Religious & $-.27^{*}$ & -.13 \\
\hline Good mental health & $-.24^{*}$ & -.13 \\
\hline Sensation-seeking & $.39+$ & .13 \\
\hline Deviant behavior & $.25^{*}$ & .15 \\
\hline Noncoital sex & & $2.50+$ \\
\hline Model concordance (0.5-1.0) & .79 & .90 \\
\hline \multicolumn{3}{|c|}{$\begin{array}{l}* P<.05 . \\
+P<.01 \text {. } \\
\mp \text { Age and dichotomous variables were not standardized because } \\
\text { their scales are interpretable in nonstandardized form. } \\
\text { § The comparison group was non-Hispanic white and races other } \\
\text { than Hispanic and African American. }\end{array}$} \\
\hline
\end{tabular}

other predictors of sexual behavior were taken into account, exposure to TV sexual content remained a strong predictor of intercourse initiation among those who were virgins at the first interview. Exposure to sexual content was also strongly predictive of progressing noncoital activity. Comparison of the $\beta$ coefficients for the sexual content variable with those for age, which was also highly predictive of sexual behavior, revealed that a 1-SD increase in exposure to sexual content on TV was equivalent in its effects on intercourse to an increase in age of 9 months $(.32 / .42=0.76$ years). Effects on noncoital behavior were equivalent to those of 17 months of aging (.31/ $.22=1.40$ years). We used the coefficients resulting from the 2 regression equations, in combination with respondents' observed standing for each predictor, to generate predicted probabilities of the outcomes at each age and for 2 different levels of TV sex exposure. The resulting estimates indicate the differential probability of having intercourse or engaging in each noncoital activity that was associated with high TV sex exposure, holding standing for other predictors constant. Figure 1 compares the predicted probability of intercourse initiation for those in the 10th percentile of exposure to TV sexual content and those in the 90th percentile. As shown, the likelihood of intercourse initiation is approximately double for the high-exposure group, across all ages studied. Figure 2 presents the predicted probabilities for breast and genital touching. The probability of initiating breast touching was $\sim 50 \%$ higher and the probability of initiating genital touching was almost double in the high-exposure group. Results for other noncoital activities were between these 2 values (data not shown).

Table 3 also shows the effects of exposure to a greater proportion of content portraying sexual behavior, rather than talk about sex. After other factors were controlled statistically, greater relative exposure to behavior remained unassociated with later sexual behavior. We also found no significant association between exposure to portrayals of sexual risk and/or safety and later sexual behavior and no association between average hours of TV viewing and sex in these multivariate models.

Many of the other respondent characteristics that were bivariate predictors of intercourse initiation remained significant in the multivariate model, including older age, having mostly older friends, lower parent education, not living with both parents, less parental monitoring, less religiosity, poor mental health, sensation-seeking personality, deviant behavior, and low school grades. Only a small subset of these factors predicted noncoital activity in the multivariate model. Other than viewing sexual content, older age and less parental monitoring predicted advancing noncoital activity.

The effects of TV viewing were largely similar across demographic groups, with a few key exceptions. In the model predicting intercourse, we noted 1 significant interaction (regression coefficient = $-.53, P<.05$ ). Although neither simple effect was significant in follow-up stratified models, there was a trend such that African Americans with high levels 
Fig 1. Predicted probabilities of intercourse initiation among virgins exposed to high versus low levels of TV sexual content.

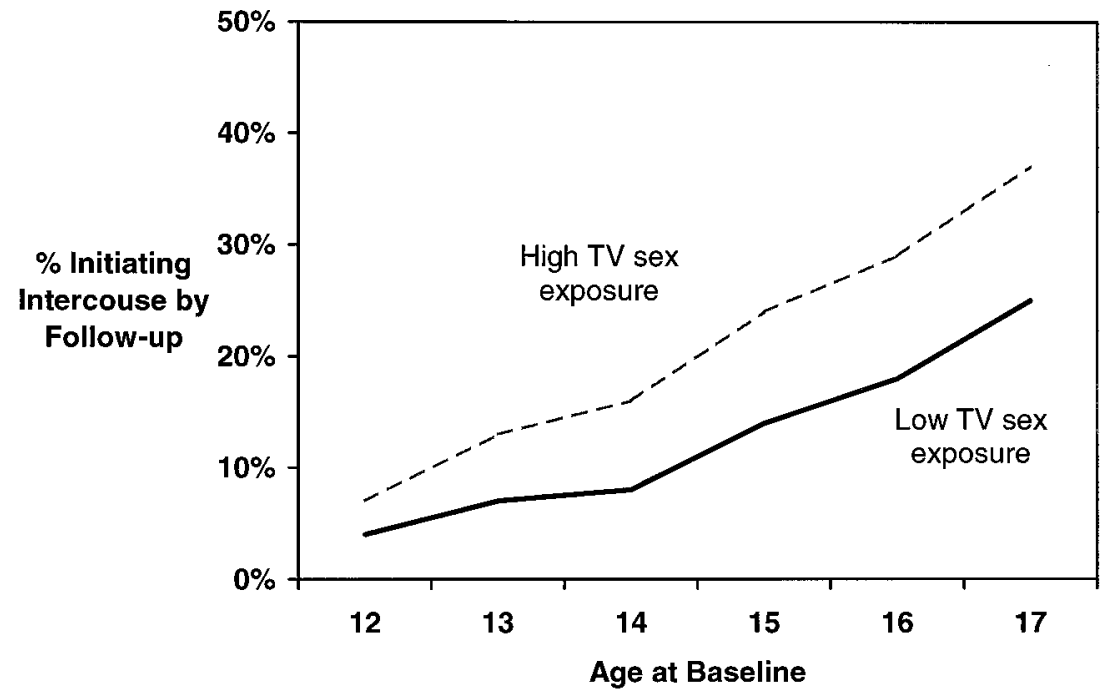

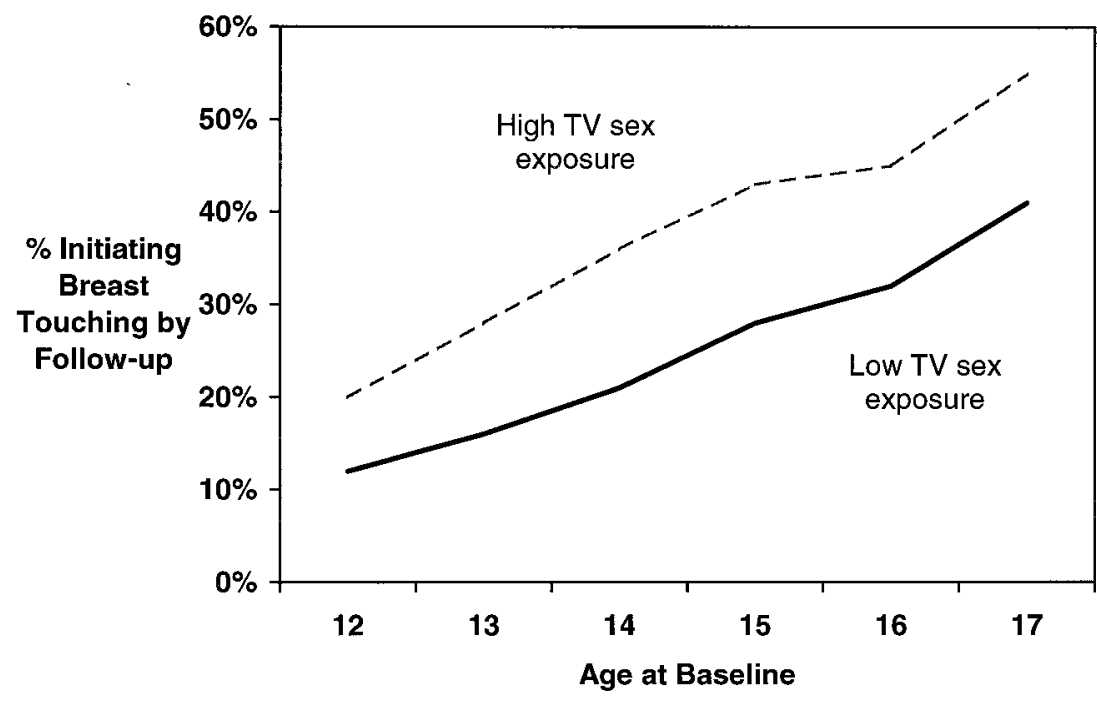

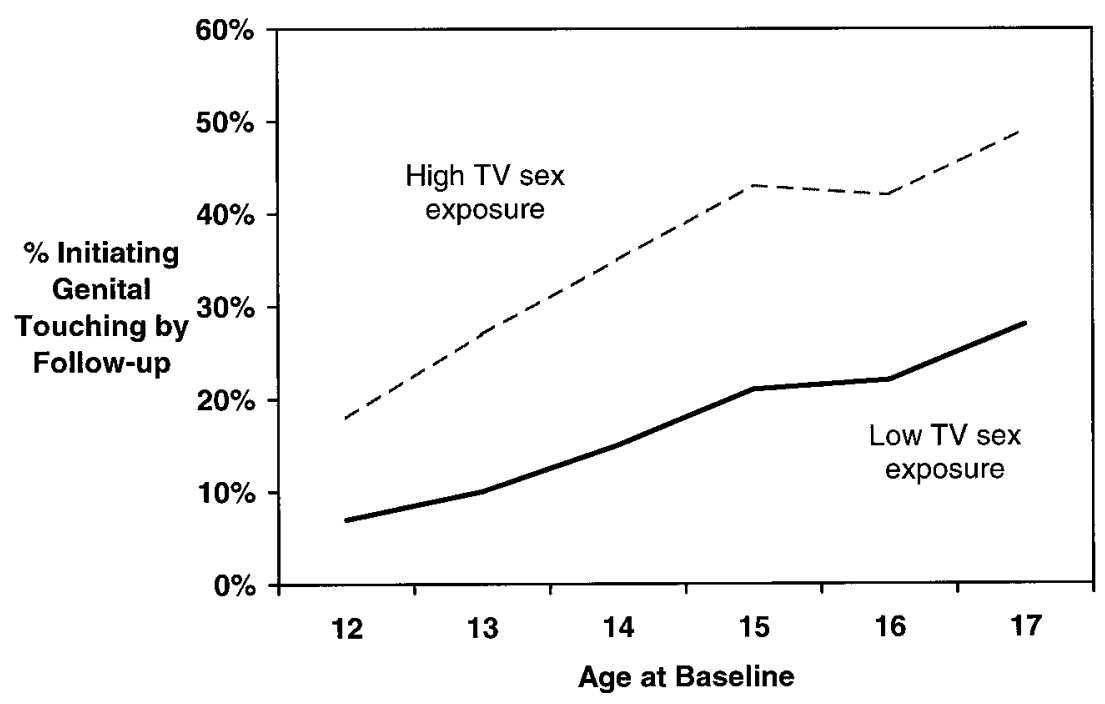

Fig 2. Predicted probabilities of noncoital advancement among those exposed to high versus low levels of TV sexual content. of exposure to sexual risk and safety portrayals were less likely to have intercourse, whereas the sexual behavior of individuals from all other races combined was not related to such exposure (African American: regression coefficient $=-.67$; all other races: regression coefficient $=.00)$. This race interaction was also significant for noncoital activity (regression coefficient $=-.21, P<.05$ ). African Americans were less likely to advance their noncoital activity level with exposure to sexual risk and safety 
portrayals (regression coefficient $=-.71, P<.01$ ), whereas changes in the activity levels of other races were not related to such exposure (regression coefficient $=-.03$, not significant).

The model predicting noncoital sex also produced a significant interaction between gender and total hours of TV viewing (regression coefficient $=.26$, $P<.05)$. More time spent watching TV delayed noncoital activity among male subjects but had no effect among female subjects (male: regression coefficient $=-.31, P<.05$; female: regression coefficient $=.08$, not significant).

\section{DISCUSSION}

We observed substantial associations between the amount of sexual content viewed by adolescents and advances in their sexual behavior during the subsequent year. Youths who viewed 1 SD more sexual content than average behaved sexually like youths who were 9 to 17 months older but watched average amounts of sex on TV. This effect is not insubstantial. Predicted probabilities showed that watching the highest levels of sexual content effectively doubled the next-year likelihood of initiating intercourse and greatly increased the probability of advancing 1 level in noncoital activity. In other words, after adjustment for other differences between high and low viewers of sexual content, 12-year-olds who watched the highest levels of this content among youths their age appeared much like youths 2 to 3 years older who watched the lowest levels of sexual content among their peers. The magnitude of these results are such that a moderate shift in the average sexual content of adolescent TV viewing could have substantial effects on sexual behavior at the population level.

It is noteworthy that the association between viewing sexual content and intercourse initiation appeared to be much stronger before our introduction of covariates to the model. The finding that these factors reduce the effects of TV viewing on behavior demonstrates the importance of including such controls in future research. The majority of factors we examined in our work predicted both viewing of sexual content and advances in sexual behavior. Nonetheless, when we controlled statistically for these associations, the relationship between exposure to TV sex and later sexual behavior remained substantial, indicating that it could not be explained by any of the variables in our study. Relationships between viewing sexual content and advancing sexual behavior were not attributable to the effects of developing sexual behavior on selective viewing of sexual content. Our analyses controlled for adolescents' level of sexual activity at baseline, rendering an explanation of reverse causality for our findings implausible.

This result replicates and extends the findings of Brown and Newcomer. ${ }^{18}$ Those authors found the same pattern of association with a sex-heavy TV diet in their research, but they could not clearly eliminate third-variable and reverse-causality explanations. We also extend their result by showing that it holds for noncoital sexual behaviors, across an age span of 5 years.
We did not find an association between sexual behavior and average hours of TV viewing. This fails to replicate the findings of Peterson et al. ${ }^{19}$ Of course, the TV environment has changed since that study, with more available channels and thus more program choices and diversity. This makes prediction of content effects on the basis of amount of exposure more difficult today than in the past. Our result also undercuts cultivation theory, which suggests that TV content is homogeneous enough that overall viewing should predict sexual outcomes. Although the process of media cultivation may well take place, content is not as uniform as it was when the theory was proposed, which perhaps explains why the prediction was not supported. We observed a nonsignificant but suggestive negative coefficient for hours of viewing predicting noncoital sex (with other variables controlled). This would be consistent with a "babysitter effect" often attributed to TV, in which youths who spend more time viewing have less time to engage in problem behaviors.

We obtained the predicted relationship between exposure to portrayals of sexual risk or the need for sexual safety and delay of sexual behavior, but only among African American youths. It would probably be a mistake to conclude that this kind of content has no effect on youths of other races, however, on the basis of this evidence. As we noted, sexual content that portrays the possible negative outcomes of having sex is extremely rare and typically receives little emphasis when it is included. ${ }^{10}$ This limited the power of our statistical tests, making it less likely that we would observe an effect. Studies of youths exposed to larger doses of risk portrayals must be conducted before a clear conclusion can be reached in this matter. The fact that an association between viewing of sexual risks and delay of activity was observed among African Americans indicates that they are more strongly affected by such portrayals. It may be that African Americans are more likely to use TV as a source of sexual information than are other groups. Other studies suggested that African Americans watch more TV and interpret sexual content differently. 9,32 However, we know of no theory that explains those findings, or our own, and we think that this is an important area for future research.

Portrayals of sexual talk and sexual behavior appear to have similar effects on youths. This is not surprising from a theoretical standpoint. Social learning theory posits that information is gleaned from what others say about a behavior as well as what they do, because both indicate social approval or disapproval of the activity in question. It apparently makes little difference whether a TV show presents people talking about whether they have sex or shows them actually having sex. Both affect perceived norms regarding sex, and thus sexual behavior.

Finally, we found that a number of factors were predictors of the transition to intercourse, consistent with previous work. However, there were fewer multivariate predictors of noncoital sex. This indicates the need for greater study of such behaviors, which may be increasingly common among adoles- 
cents as they struggle to become sexually active in a way that they perceive to be safe and/or preserving of virginity. ${ }^{33}$

A limitation of this research was our inability to control for adolescent interest in sex or sexual readiness before TV viewing. Youths who are considering coital or noncoital activities that they have not yet enacted may watch more sex on TV (eg, to get information or to satisfy desires). They may subsequently engage in these sexual activities sooner but as a result of their higher levels of interest, not as a result of their TV exposure. It was not possible for us to test for this alternative interpretation of our results with only 2 waves of data. However, it is likely that much of this sexual interest tendency was recorded with the covariates in our models, because they are known predictors of sexual initiation. Moreover, ancillary analyses indicate that sexual attitudes, beliefs, and behaviors at baseline jointly predict only a small portion of the variance in viewing sexual content at the follow-up assessment (results available from the authors on request).

Other limitations appear to be based on the sensitive nature of our research topic. Although rates of intercourse were within the expected range at the follow-up evaluation, they were somewhat low at the baseline evaluation, which suggests that some participants were not initially honest about their sexual experience. In addition, analyses assessing the characteristics of the 175 participants who refused to answer sexual behavior questions indicated that these youths watched more TV and were more involved viewers. They also watched marginally less sex on TV. This suggests that those who were dropped from analyses because of missing sex data might have been more vulnerable to the effects of sexual content, because TV played a greater role in their lives and they were more sheltered from sexual content. This might also be true of subjects who were eligible for the study but declined to be interviewed at all. Those individuals were probably more sexually conservative than the analytic sample. Although the effect of this factor on our findings cannot be determined, it is probable that omitting this group restricted the range and/or variability of the prediction and outcome variables and might have produced an underestimate of the relationship between viewing of sexual content and later sexual behavior.

Finally, although TV accounts for more of children's time than any other medium, ${ }^{9}$ the sexual content in films, music, and magazines is also likely to hasten sexual advancement. Therefore, it will be important to address these other contributions to sexual socialization in both future research and interventions. Indeed, we should note that, because TV viewing of sexual content is probably related to exposure to sex in other media, our results may reflect at least partially the influence of music, magazines, or movies. Research assessing the independent contribution of each medium to adolescent sexuality is needed.

With these limitations in mind, our findings have clear implications. Reducing the amount of sexual talk and behavior on TV or the amount of time that adolescents are exposed to this content is likely to appreciably delay the initiation of both coital and noncoital sexual activities. Increasing the percentage of portrayals of sexual risk and safety, relative to other sexual content, might also inhibit early sexual activity, particularly among African American youths. However, reducing exposure to sexual content on TV may be difficult. An option that does not require altering TV content or adolescents' viewing habits has met with some success in other areas. Parents who view violent programs with their children and discuss their own beliefs regarding the behavior depicted may be able to reduce the effects of positively portrayed aggressive content on their children's behavior. ${ }^{34}$ This process may also help limit the negative effects of sexual portrayals that do not contain risk information. Our recommendation is in line with that of the American Academy of Pediatrics, which suggests that pediatricians encourage family discussions of the media and its effect on sexual behavior. Our research offers only limited insight into the best method for ameliorating the effects of TV sexual content on youth behavior but clearly indicates the need to do so.

\section{ACKNOWLEDGMENTS}

This research was funded by grant HD38090-02 from the National Institute of Child Health and Human Development. data.

We thank Nielsen Media Research for providing TV ratings

\section{REFERENCES}

1. LeVay S, Valente S. Human Sexuality. Sunderland, MA: Sinauer Associates; 2003

2. Centers for Disease Control and Prevention. Trends in sexual risk behaviors among high school students: United States, 1991-2001. MMWR Morb Mortal Wkly Rep. 2002;51:856-859

3. National Campaign to Prevent Teen Pregnancy. With One Voice 2002: America's Adults and Teens Sound Off About Teen Pregnancy. Washington, DC: The National Campaign to Prevent Teen Pregnancy; 2002

4. Institute of Medicine. The Hidden Epidemic: Confronting Sexually Transmitted Diseases. Washington, DC: National Academy Press; 1997

5. Singh S, Darroch JE. Trends in sexual activity among adolescent American women: 1982-1995. Fam Plann Perspect. 1999;31:212-219

6. Koyle P, Jensen L, Olsen J. Comparison of sexual behaviors among adolescents having an early, middle and late first intercourse experience. Youth Soc. 1989;20:461-476

7. Delameter J. The social control of sexuality. Annu Rev Sociol. 1981;7: 263-290

8. Nathanson C. Dangerous Passage: The Social Control of Sexuality in Women's Adolescence. Philadelphia, PA: Temple University Press; 1991

9. Roberts DF, Foehr UG, Rideout VJ, Brodie M. Kids \& Media @ the New Millennium: A Kaiser Family Foundation Report: A Comprehensive National Analysis of Children's Media Use: Executive Summary. Menlo Park, CA: The Henry J Kaiser Family Foundation; 1999

10. Kunkel D, Eyal K, Biely E, et al. Sex on TV3: A Biennial Report to the Kaiser Family Foundation. Menlo Park, CA: The Henry J. Kaiser Foundation; 2003. Available at: www.kff.org/entmedia/loader.cfm?url=/ commonspot/security/getfile.cfm\&PageID=14209. Accessed July 20, 2004

11. Gerbner G, Gross M, Morgan L, Signorielli N. Living with television: the dynamics of the cultivation process. In: Bryant J, Zillman D, eds. Perspectives on Media Effects. Hillsdale, NJ: Lawrence Erlbaum Associates; 1986:17-40

12. Bandura A. Social Foundations of Thought and Action: A Social Cognitive Theory. Englewood Cliffs, NJ: Prentice Hall; 1986

13. American Psychological Association. Violence and Youth: Psychology's Response. Washington, DC: American Psychological Association; 1993

14. Strouse J, Buerkel-Rothfus N. Media exposure and the sexual attitudes and behaviors of college students. In: Greenberg B, Brown JD, BuerkelRothfus N, eds. Media, Sex and the Adolescent. Cresskill, NJ: Hampton Press;1993 
15. Ward L. Does television exposure affect emerging adults' attitudes and assumptions about sexual relationships? Correlational and experimental confirmation. J Youth Adolesc. 2002;31:1-15

16. Courtright J, Baran S. The acquisition of sexual information by young people. Journalism Q. 1980;57:107-114

17. Buerkel-Rothfuss N, Strouse J. Media exposure and perceptions of sexual behaviors: the cultivation hypothesis moves to the bedroom. In: Greenberg B, Brown J, Buerkel-Rothfuss N, eds. Media, Sex and the Adolescent. Cresskill, NJ: Hampton Press;1993

18. Brown JD, Newcomer SF. Television viewing and adolescents' sexual behavior. J Homosex. 1991;21:77-91

19. Peterson JL, Moore KA, Furstenberg FF Jr. Television viewing and early initiation of sexual intercourse: is there a link? J Homosex. 1991;21:93-118

20. Chunovic L. One Foot on the Floor: The Curious Evolution of Sex on Television From "I Love Lucy" to "South Park." New York, NY: TV Books; 2000

21. Santelli JS, Lindberg LD, Abma J, McNeely CS, Resnick M. Adolescent sexual behavior: estimates and trends from four nationally representative surveys. Fam Plann Perspect. 2000;32:156-165, 194

22. Miller K, Clark L, Wendell D, et al. Adolescent heterosexual experience: a new typology. J Adolesc Health. 1997;20:179-186

23. Kosterman R, Hawkins J, Guo J, Catalano R, Abbott R. The dynamics of alcohol and marijuana initiation: patterns and predictors of first use in adolescence. Am J Public Health. 2000;90:360-366

24. Hanson S, Myers D, Ginsburg A. The role of responsibility and knowl edge in reducing teenage out-of-wedlock childbearing. J Marriage Fam. 1987;49:241-256

25. Ware JE Jr, Sherbourne CD. The MOS 36-item short-form health survey (SF-36). I. Conceptual framework and item selection. Med Care. 1992;30: $473-483$

26. Rosenberg M. Society and the Adolescent Self-Image. Princeton, NJ: Princeton University Press; 1965

27. Collins RL, Ellickson PL, Bell RM. Simultaneous polydrug use among teens: prevalence and predictors. J Subst Abuse. 1998;10:233-253

28. Zuckerman M. Notes and shorter communications: item revisions in the Sensation Seeking Scale Form V (SSS-V). Pers Individ Diff. 1996;20:515

29. Kish L. Survey Sampling. Oxford, England: Wiley; 1965

30. Little R, Rubin D. Statistical Analysis With Missing Data. New York, NY: Wiley; 1987

31. Kotz S, Johnson NL, Read CB. Encyclopedia of Statistical Sciences. New York, NY: Wiley; 1986

32. Brown JD, Schulze L. The effects of race, gender, and fandom on audience interpretations of Madonna's music videos. J Commun. 1990; 40:88-102

33. Remez L. Oral sex among adolescents: is it sex or is it abstinence? Fam Plann Perspect. 2000;32:298-304

34. Donnerstein E, Slaby R, Eron L. The mass media and youth aggression. In: Eron LD, Gentry JH, Schlegel P, eds. Reason to Hope: A Psychosocial Perspective on Violence and Youth. Washington, DC: American Psychological Association; 1994:219-250 